\title{
A cumulative score based on preoperative fibrinogen and the neutrophil-lymphocyte ratio to predict outcomes in resectable gastric cancer
}

This article was published in the following Dove Press journal:

Cancer Management and Research

\section{Xuechao Liu ${ }^{1,2, *}$ \\ Zhimin Liu',2,* \\ Enzi Lin 3 ,* \\ Yingbo Chen ${ }^{1,2}$ \\ Xiaowei Sun ${ }^{1,2}$ \\ Zhiwei Zhou ${ }^{1,2}$}

'Sun Yat-sen University Cancer Center, State Key Laboratory of Oncology in South China, Collaborative Innovation Center for Cancer Medicine, Guangzhou, 510060, People's Republic of China; ${ }^{2}$ Department of Gastric Surgery, Sun Yat-sen University Cancer Center, 510060, Guangzhou, People's Republic of China; ${ }^{3}$ Surgical Oncology Session No.I, Cancer Hospital of Shantou University Medical College, Shantou, 5I504I, People's Republic of China

*These authors contributed equally to this work
Correspondence: Zhiwei Zhou;

Xiaowei Sun

Department of Gastric Surgery, Sun

Yat-sen University Cancer Center, 65I

East Dongfeng Road, Guangzhou, 510060,

Guangdong, People's Republic of China

Tel +86 208734 3I 23; +862087343910

Email zhouzhw@sysucc.org.cn;

sunxw@sysucc.org.cn
Background: Recent studies have revealed that preoperative fibrinogen and the neutrophil-lymphocyte ratio (NLR) are associated with poor outcome in gastric cancer (GC). We aimed to evaluate whether the fibrinogen and the NLR score had a consistent prognostic value for resectable GC. Methods: We analyzed 1,293 consecutive patients who underwent curative surgery for GC. The F-NLR score was 2 for patients with hyperfibrinogenemia ( $>400 \mathrm{mg} / \mathrm{dL})$ and elevated $\operatorname{NLR}(\geq 5.0), 1$ for those with one abnormal index, and 0 for those with no abnormal indices.

Results: We found that higher F-NLR scores were associated with larger tumor size, deeper tumor invasion and more lymph node metastasis (all $P<0.05$ ). In a multivariate analysis, F-NLR independently predicted postoperative survival $(P<0.001)$. When stratified by tumor-nodemetastasis (TNM) stage, the prognostic value of F-NLR was still maintained for stages I-II $(P<0.001)$ and stage III $(P=0.003)$. Of note, F-NLR also effectively stratified overall survival (OS) irrespective of age, adjuvant chemotherapy administration, tumor location and histological grade (all $P<0.05$ ). Furthermore, F-NLR and TNM stratified 5-year OS from 61\% (F-NLR 0) to $15 \%$ (F-NLR 2) and from $92 \%$ (stage I) to $37 \%$ (stage III), respectively. Utilizing both F-NLR and TNM, 5-year OS ranged from 93\% (F-NLR 0, TNM I) to 6\% (F-NLR 2, TNM III). Conclusion: The F-NLR score independently predicts outcomes in GC patients after curative surgery. Therefore, it should be implemented in routine clinical practice for identifying more high-risk patients.

Keywords: F-NLR score, gastric cancer, prognosis, adjuvant chemotherapy, curative surgery

\section{Introduction}

Although the incidence of gastric cancer (GC) is decreasing, it remains one of the main causes of cancer-related death in the world, with a high rate of tumor recurrence and distant metastasis. ${ }^{1,2}$ Early detection is important. However, early-stage GC is often clinically silent with nonspecific symptoms. ${ }^{3}$ Although there have been massive strides in surgery and postoperative treatment, the clinical outcomes for GC patients remain poor with high rates of late diagnosis. ${ }^{4,5}$ Thus, it is of vital importance to identify independent prognostic factors to select patients for tailored treatments.

Over the past decades, numerous studies have revealed that several systemic inflammation scores, such as the neutrophil-lymphocyte ratio (NLR) and platelet-lymphocyte ratio (PLR), have important prognostic significance in various malignancies. ${ }^{6-8} \mathrm{~A}$ recent meta-analysis of ten studies provided concrete evidence that high NLR might be correlated with poor outcome in GC. ${ }^{9}$ In addition, fibrinogen, a $340 \mathrm{kD}$ liver glycoprotein, has been shown to play an essential role in both systemic inflammatory responses and 
tumor progression. ${ }^{10,11}$ Many investigators have demonstrated the clinical utility of fibrinogen as a prognostic predictor in various types of malignancies. ${ }^{12,13}$ Recently, a new scoring system combining preoperative fibrinogen and the NLR (F-NLR) has been demonstrated as a promising prognostic marker for esophageal squamous cell carcinoma. ${ }^{14,15}$ However, the prognostic role of F-NLR in GC remains unclear.

In the current study, the clinical utility of F-NLR was determined in a large cohort of GC patients after curative surgery.

\section{Patients and methods Patients' characteristics}

We reviewed a clinical database of 1,293 consecutive GC patients after curative surgery at Sun Yat-sen University Cancer Center from October 2000 to September 2012. Our study protocol was conducted in accordance with the standards of the Declaration of Helsinki and approved by the ethical committee of Sun Yat-sen University Cancer Center. Informed consent was deemed unnecessary by the ethical committee, and all information was anonymous. The eighth edition of the tumor-node-metastasis (TNM) criteria was used for tumor staging, and other information was gathered from medical records.

The inclusion criteria of this study were patients who had the following: 1) histologically proven gastric adenocarcinoma, 2) complete data on clinicopathological features and outcome, 3) no neoadjuvant therapy, 4) no multiple primary synchronous malignant tumors and 5) no clinical evidence of infection or other inflammatory disease for at least 1 month before surgery.

Blood specimens were obtained within 1 week before surgery. Plasma fibrinogen concentrations were measured using a CS5100 coagulation analyzer (Sysmex Co., Kobe, Japan). Neutrophils, lymphocytes, platelet counts, and mean platelet volume were evaluated using the XN2000 automated hematology analyzer (Sysmex Co.). By multidisciplinary discussion, 5-fluorouracil-based adjuvant chemotherapy or combined treatment was routinely delivered to stage II-III GC patients without marked comorbidities that would preclude chemotherapy.

\section{Follow-up}

The routine follow-up scheme included a clinical review approximately 4 weeks post operation, followed by a complete history, physical examination, blood routine examination, gastroscopy and chest/abdominal/pelvic computerized tomography (CT) imaging, all routinely performed every
3 months during the first postoperative year and every 6 months afterward. The final follow-up date for our study was June 2015. Overall survival (OS) was calculated as the interval from the initial surgery until death or last follow-up.

\section{Calculation of prognostic scores}

The common cutoff threshold for plasma fibrinogen level was $400 \mathrm{~g} / \mathrm{L}$ as previously described. ${ }^{16,17}$ Similarly, the NLR and PLR also used widely accepted thresholds. ${ }^{18-21}$ The F-NLR was calculated as follows: an F-NLR score of 2, with hyperfibrinogenemia ( $>400 \mathrm{mg} / \mathrm{dL}$ ) and elevated NLR ( $\geq 5.0)$; an F-NLR score of 1, with one abnormal index; an F-NLR score of 0 , with no abnormal indices.

\section{Statistical analysis}

Relationships between the F-NLR score and categorical clinicopathological features were assessed using a two-tailed chisquared test. Differences between the OS rate generated by the Kaplan-Meier curves were compared using the log-rank test. Prognostic factors were determined by the Cox proportional hazards model. All data were analyzed using SPSS version 19.0 (IBM Corporation, Armonk, NY, USA). A two-sided $P$-value of $<0.05$ was regarded as statistically significant. All data have been recorded at Sun Yat-sen University Cancer Center for future reference (number RDDA2017000336).

\section{Results}

Among the 1,293 patients ( 882 men), the median age was 59 (range 19-89) years (Table 1). The median duration of follow-up was 35 months. The mean preoperative plasma fibrinogen level ( \pm SD) was $330 \pm 91 \mathrm{mg} / \mathrm{dL}$ (range 64-802). Patients were classified into two independent groups based on the cutoff threshold of fibrinogen and NLR (low $\leq 400 \mathrm{mg}$ / $\mathrm{dL}$ or high $>400 \mathrm{mg} / \mathrm{dL}$ and low $<5$ or high $\geq 5$, respectively). Patients with hyperfibrinogenemia and elevated NLR had a poorer OS than those with low plasma fibrinogen levels and NLR $(P<0.001$ and $P<0.001$, respectively; Figure 1$)$. In addition, further analysis showed that fibrinogen was positively correlated with NLR $(P<0.001)$. Based on the criteria of the F-NLR score, patients were divided into three independent groups (F-NLR 0, n=986; F-NLR 1, n=279; F-NLR 2, n=28).

The correlation between F-NLR score and clinicopathological features is summarized in Table 2. Higher F-NLR scores were more likely found in males $(P=0.015)$ and were associated with older age $(P<0.001)$, larger tumor size $(P<0.001)$, tumor location in the upper third of the stomach $(P=0.005)$, elevated PLR $(P<0.001)$, deeper tumor invasion $(P<0.001)$ and more lymph node metastasis $(P=0.003)$. 
Table I The clinicopathological features of I,293 GC patients

\begin{tabular}{|c|c|}
\hline Features & Patients, n (\%) \\
\hline \multicolumn{2}{|l|}{ Age (years) } \\
\hline$<60$ & $685(53.0)$ \\
\hline$\geq 60$ & $608(47.0)$ \\
\hline \multicolumn{2}{|l|}{ Sex } \\
\hline Female & $4 I I(3 I .8)$ \\
\hline Male & $882(68.2)$ \\
\hline \multicolumn{2}{|l|}{ Tumor size $(\mathrm{cm})$} \\
\hline$<5$ & $720(55.7)$ \\
\hline$\geq 5$ & $573(44.3)$ \\
\hline \multicolumn{2}{|l|}{ Tumor location } \\
\hline Upper third & $50 \mathrm{I}(38.7)$ \\
\hline Middle third & $268(20.7)$ \\
\hline Lower third & $524(40.5)$ \\
\hline \multicolumn{2}{|l|}{ Histological grade } \\
\hline Well differentiated & $219(16.9)$ \\
\hline Poorly differentiated & $\mathrm{I}, 074(83 . \mathrm{l})$ \\
\hline \multicolumn{2}{|l|}{ NLR } \\
\hline$<5.0$ & $\mathrm{I}, 22 \mathrm{I}(94.4)$ \\
\hline$\geq 5.0$ & $72(5.6)$ \\
\hline \multicolumn{2}{|l|}{ Fibrinogen (mg/dL) } \\
\hline$\leq 400$ & $\mathrm{I}, 030(79.7)$ \\
\hline$>400$ & $263(20.3)$ \\
\hline \multicolumn{2}{|l|}{ PLR } \\
\hline$<150$ & $792(61.3)$ \\
\hline$\geq 150$ and $\leq 300$ & $438(33.9)$ \\
\hline$>300$ & $63(4.9)$ \\
\hline \multicolumn{2}{|l|}{ MPV (fL) } \\
\hline$<10$ & 981 (77.1) \\
\hline$\geq 10$ & $292(22.9)$ \\
\hline \multicolumn{2}{|l|}{ F-NLR } \\
\hline 0 & $986(76.3)$ \\
\hline 1 & $279(21.6)$ \\
\hline 2 & $28(2.2)$ \\
\hline \multicolumn{2}{|l|}{ TNM stage } \\
\hline I & $218(16.9)$ \\
\hline II & $322(24.9)$ \\
\hline III & $753(58.2)$ \\
\hline \multicolumn{2}{|l|}{ Adjuvant chemotherapy } \\
\hline No & $498(38.5)$ \\
\hline Yes & $795(6 \mid .5)$ \\
\hline
\end{tabular}

Note: F-NLR, combination of fibrinogen and the NLR.

Abbreviations: GC, gastric cancer; MPV, mean platelet volume; NLR neutrophillymphocyte ratio; PLR, platelet-lymphocyte ratio; TNM, tumor-node-metastasis.

In a univariate survival analysis, eight clinicopathological parameters were significant predictors of survival (Table 3). After excluding the related parameters, the multivariate analysis identified the F-NLR score as an independent predictor of outcome (HR for F-NLR 1 and F-NLR 2: 1.274, 95\% CI $1.043-1.555$ and $2.228,95 \%$ CI $1.423-3.487$, respectively; $P<0.001)$.

When stratified by TNM stage, the prognostic value of F-NLR was still maintained for stages I-II $(P<0.001)$ and stage III ( $P=0.003$; Figure 2). Furthermore, F-NLR also effectively stratified OS irrespective of age, adjuvant chemotherapy administration, tumor location and histological grade (all $P<0.05$; Figure 3). Of note, F-NLR and TNM stratified 5 -year OS from $61 \%$ (F-NLR 0 ) to $15 \%$ (F-NLR 2) and from $92 \%$ (stage I) to $37 \%$ (stage III), respectively, whereas when utilizing both F-NLR and TNM, 5-year OS ranged from 93\% (F-NLR 0, TNM I) to 6\% (F-NLR 2, TNM III; Table 4).

\section{Discussion}

The results of the current study show that F-NLR, a newly proposed cumulative score, was an independent prognostic predictor in GC. Of note, its prognostic significance was still maintained in subgroups, including patients diagnosed with TNM stages I-II or stage III, as well as patients who did or did not receive adjuvant chemotherapy. Furthermore, F-NLR also effectively stratified OS irrespective of age, tumor location and histological grade.

Over the past decades, it has become widely accepted that cancer and inflammation are linked. ${ }^{22,23}$ Indeed, regardless of stage, the maintenance and development of the systemic inflammatory responses have been consistently linked to poorer prognosis. ${ }^{24,25}$ Although many organ systems act in the activation of systemic inflammatory responses, the common measurements of the magnitude of activation are circulating cellular components and acute-phase proteins. In particular, several common peripheral blood-derived inflammationbased scores, such as the Glasgow Prognostic Score (GPS), NLR, and PLR, have been demonstrated as promising prognostic predictors in various malignancies. ${ }^{26-28}$ However, the measurement of serum C-reactive protein was not routinely taken in our hospital before 2005, and therefore, the prognostic value of GPS was not examined in the current study.

In addition, much attention has been paid to the association between hypercoagulation and malignancy progression in recent years. ${ }^{29,30}$ Accumulating evidence indicates that the activation of the coagulation cascade plays a key pathophysiological role during tumor progression. ${ }^{31}$ Fibrinogen, as an important component of the coagulation cascade, has been demonstrated as an essential regulator in both the systemic inflammatory response and tumor progression. First, fibrinogen provides a stable framework for the tumor extracellular matrix, which may promote tumor angiogenesis and enhance tumor cell adhesion, migration and invasion. ${ }^{32}$

Moreover, as an acute-phase protein, fibrinogen is released in response to infection or systemic inflammation. It may mediate the initial adhesion of leukocytes to endothelial cells and the release of pro-inflammatory cytokines, thereby leading to tumor cell proliferation and 


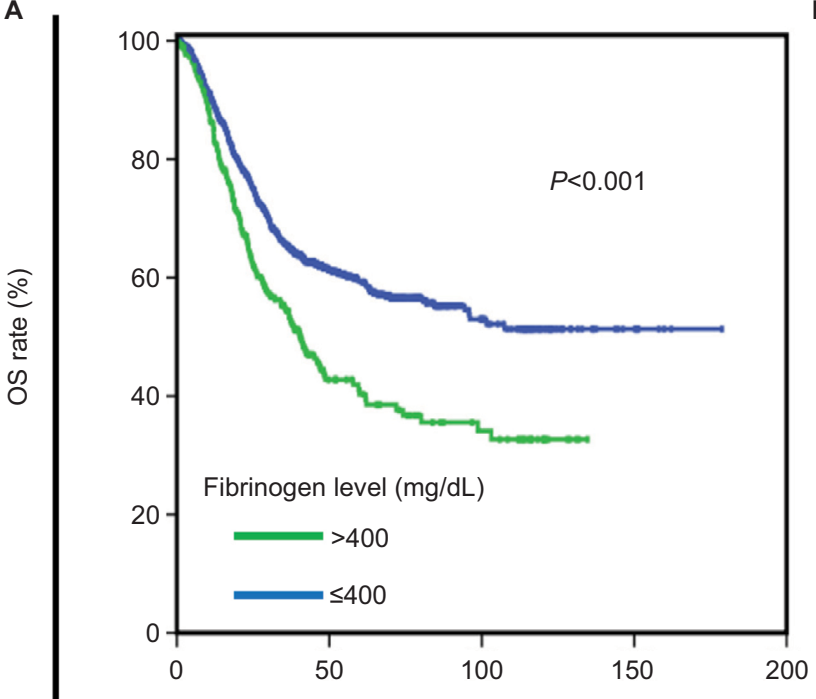

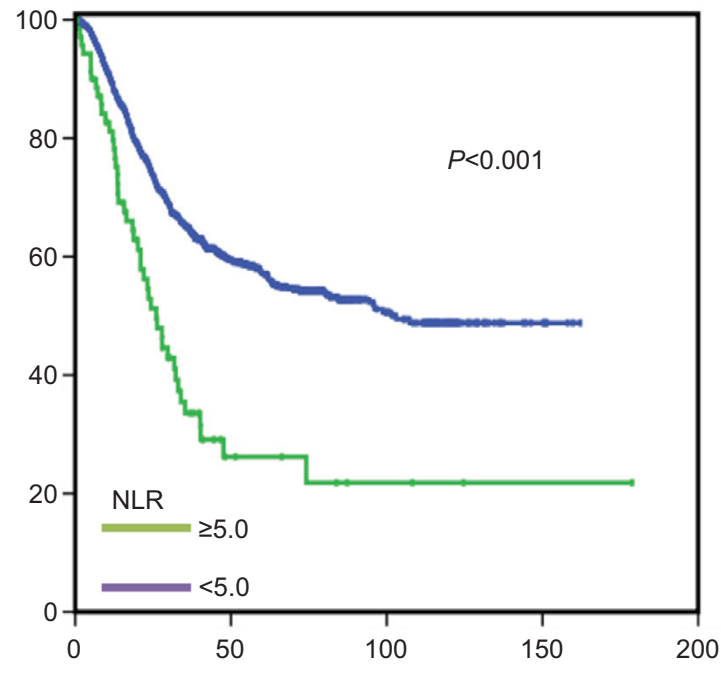

Time after surgery (months)

Figure I Kaplan-Meier survival curves according to preoperative fibrinogen (A) and the NLR (B).

Abbreviations: NLR, neutrophil-lymphocyte ratio; OS, overall survival.

Table 2 The clinicopathological characteristics stratified by the F-NLR score

\begin{tabular}{|c|c|c|c|c|}
\hline Characteristics & $\begin{array}{l}\text { F-NLR } 0 \\
(n=986)\end{array}$ & $\begin{array}{l}\text { F-NLR I } \\
(n=279)\end{array}$ & $\begin{array}{l}\text { F-NLR } 2 \\
(n=28)\end{array}$ & P-value* \\
\hline Age (years) & & & & $<0.001$ \\
\hline$<60$ & 558 & 118 & 9 & \\
\hline$\geq 60$ & 428 & 161 & 19 & \\
\hline Sex & & & & 0.015 \\
\hline Female & 329 & 79 & 3 & \\
\hline Male & 657 & 200 & 25 & \\
\hline Tumor size $(\mathrm{cm})$ & & & & $<0.001$ \\
\hline$<5$ & 606 & 105 & 9 & \\
\hline$\geq 5$ & 380 & 174 & 19 & \\
\hline Tumor location & & & & 0.005 \\
\hline Upper third & 357 & 128 & 16 & \\
\hline Middle third & 212 & 49 & 7 & \\
\hline Lower third & 417 & 102 & 5 & \\
\hline Histological grade & & & & 0.093 \\
\hline Well differentiated & 155 & 57 & 7 & \\
\hline Poorly differentiated & 831 & 222 & 21 & \\
\hline PLR & & & & $<0.001$ \\
\hline$<150$ & 678 & 113 & I & \\
\hline$\geq 150$ and $\leq 300$ & 285 & 138 & 15 & \\
\hline$>300$ & 23 & 28 & 12 & \\
\hline MPV (fL) & & & & 0.069 \\
\hline$<10$ & 733 & 225 & 23 & \\
\hline$\geq 10$ & 237 & 51 & 4 & \\
\hline Tumor depth & & & & $<0.001$ \\
\hline TI & 142 & 13 & 0 & \\
\hline $\mathrm{T} 2$ & 120 & 19 & I & \\
\hline T3 & 211 & 64 & 8 & \\
\hline $\mathrm{T} 4$ & 513 & 183 & 19 & \\
\hline Lymph node & & & & 0.003 \\
\hline No & 347 & 66 & 4 & \\
\hline $\mathrm{NI}$ & 148 & 49 & 8 & \\
\hline N2 & $17 \mid$ & 51 & 5 & \\
\hline N3 & 320 & 113 & II & \\
\hline \multicolumn{5}{|c|}{$\begin{array}{l}\text { Notes: F-NLR, combination of fibrinogen and the NLR. *Values in bold refer to } \\
P \text {-value with significance }(P<0.05) \text {. }\end{array}$} \\
\hline
\end{tabular}

progression. ${ }^{33}$ Recent studies have presented evidence that fibrinogen correlates with tumor progression, poor response to chemotherapy and adverse clinical outcome in various types of cancer. ${ }^{12,34}$

Recently, a newly proposed scoring system (F-NLR) has garnered increasing attention in esophageal squamous cell carcinoma. Kijima et $\mathrm{al}^{14}$ proved that the F-NLR score had clinical potential as a predictor of therapeutic effects and outcomes in advanced esophageal squamous cell carcinoma. Another study, which evaluated 238 patients undergoing esophagectomy for esophageal squamous cell carcinoma, reported that the F-NLR score was a promising predictive marker of tumor progression and prognosis. ${ }^{15}$

However, in GC, the utility of the F-NLR score has not yet been systematically analyzed. Although Arigami et al ${ }^{35}$ found that the F-NLR score was an independent prognostic predictor in advanced GC, they only enrolled 68 patients with distant metastatic GC including locally advanced tumor and recurrent disease. The enrolled patients underwent nonuniform treatment, including surgery, chemotherapy or chemoradiotherapy. Because of the limited cases, they did not perform subgroup analyses to further verify the prognostic value. However, we aimed to determine whether the F-NLR score could better predict survival of patients after curative resection for stage I-III GC. Therefore, we performed a large-scale retrospective study and found that the F-NLR score could independently predict survival after curative resection of GC. As shown in Figures 1-2, the F-NLR score can effectively divide patients into three independent groups. Undoubtedly, 
Table 3 Univariate and multivariate analyses of prognostic factors in I,296 patients with GC

\begin{tabular}{|c|c|c|c|c|}
\hline \multirow[t]{2}{*}{ Factors } & \multicolumn{2}{|l|}{ Univariate analysis } & \multicolumn{2}{|l|}{ Multivariate analysis } \\
\hline & HR (95\% Cl) & $P$-value* & HR (95\% Cl) & $P$-value* \\
\hline Age (years) & & $<0.001$ & & $<0.001$ \\
\hline$<60$ & 1.00 & & 1.00 & \\
\hline$\geq 60$ & $1.490(1.252,1.774)$ & & I. 467 (I.228, I.752) & \\
\hline Sex & & 0.667 & & \\
\hline Female & 1.00 & & & \\
\hline Male & $1.042(0.864,1.256)$ & & & \\
\hline Tumor size $(\mathrm{cm})$ & & $<0.001$ & & 0.988 \\
\hline$<5$ & 1.00 & & 1.00 & \\
\hline$\geq 5$ & $1.718(1.443,2.046)$ & & $0.999(0.833,1.198)$ & \\
\hline Tumor location & & $<0.001$ & & $<0.001$ \\
\hline Upper third & 1.00 & & 1.00 & \\
\hline Middle third & $0.673(0.535,0.847)$ & 0.001 & $0.800(0.632,1.012)$ & 0.062 \\
\hline Lower third & $0.456(0.373,0.558)$ & $<0.001$ & $0.602(0.489,0.742)$ & $<0.001$ \\
\hline Histological grade & & 0.027 & & 0.032 \\
\hline Well differentiated & 1.00 & & 1.00 & \\
\hline Poorly differentiated & $1.328(1.032,1.710)$ & & $1.327(1.024,1.720)$ & \\
\hline NLR & & $<0.001$ & & \\
\hline$<5.0$ & 1.00 & & & \\
\hline$\geq 5.0$ & $2.313(1.707,3.134)$ & & & \\
\hline Fibrinogen (mg/dL) & & $<0.001$ & & \\
\hline$\leq 400$ & 1.00 & & & \\
\hline$>400$ & $1.618(1.330,1.969)$ & & & \\
\hline PLR & & 0.069 & & \\
\hline$<150$ & 1.00 & & & \\
\hline$\geq 150$ and $\leq 300$ & $1.210(1.007,1.454)$ & 0.042 & & \\
\hline$>300$ & $1.325(0.910,1.930)$ & 0.142 & & \\
\hline MPV (fL) & & 0.571 & & \\
\hline$<10$ & 1.00 & & & \\
\hline$\geq 10$ & $0.940(0.757,1.166)$ & & & \\
\hline F-NLR & & $<0.001$ & & $<0.001$ \\
\hline 0 & 1.00 & & 1.00 & \\
\hline 1 & $1.619(1.331,1.969)$ & $<0.001$ & $1.274(1.043,1.555)$ & 0.008 \\
\hline 2 & $3.326(2.139,5.172)$ & $<0.001$ & $2.228(1.423,3.487)$ & $<0.001$ \\
\hline TNM stage & & $<0.001$ & & $<0.001$ \\
\hline 1 & 1.00 & & 1.00 & \\
\hline ॥ & $3.006(1.764,5.122)$ & $<0.001$ & $2.525(1.475,4.321)$ & 0.008 \\
\hline III & II.059 (6.807, I7.967) & $<0.001$ & $9.129(5.565,14.977)$ & $<0.001$ \\
\hline Adjuvant chemotherapy & & 0.373 & & \\
\hline No & 1.00 & & & \\
\hline Yes & $0.922(0.77 \mathrm{I}, \mathrm{I} .102)$ & & & \\
\hline
\end{tabular}

Notes: F-NLR, combination of fibrinogen and the NLR. *Values in bold refer to $P$-value with significance $(P<0.05)$.

Abbreviations: GC, gastric cancer; MPV, mean platelet volume; NLR, neutrophil-lymphocyte ratio; PLR, platelet-lymphocyte ratio; TNM, tumor-node-metastasis.

F-NLR score could identify more high-risk patients than fibrinogen or NLR alone. Overall, the data suggest that the F-NLR score may provide more potent prognostic value than the individual indices. Furthermore, higher F-NLR scores were associated with larger tumor size, deeper tumor invasion, and lymph node metastasis. This observation was in line with previous studies that indicated that the F-NLR score was significantly correlated with tumor progression and aggressive biological behavior. ${ }^{14,15}$ In subgroup analyses, the F-NLR score still exhibited potential prognostic value, as shown in Figures 2 and 3. Indeed, our conclusions have been strengthened by the subgroup analyses. Moreover, the combination of F-NLR and TNM stage increased the range of survival compared to either F-NLR or TNM alone. Therefore, the inclusion of some valuable clinicopathological features as additional prognostic factors in TNM staging does not negate the role of host characteristics, such as the F-NLR score, in effective prognostic stratification. Indeed, 


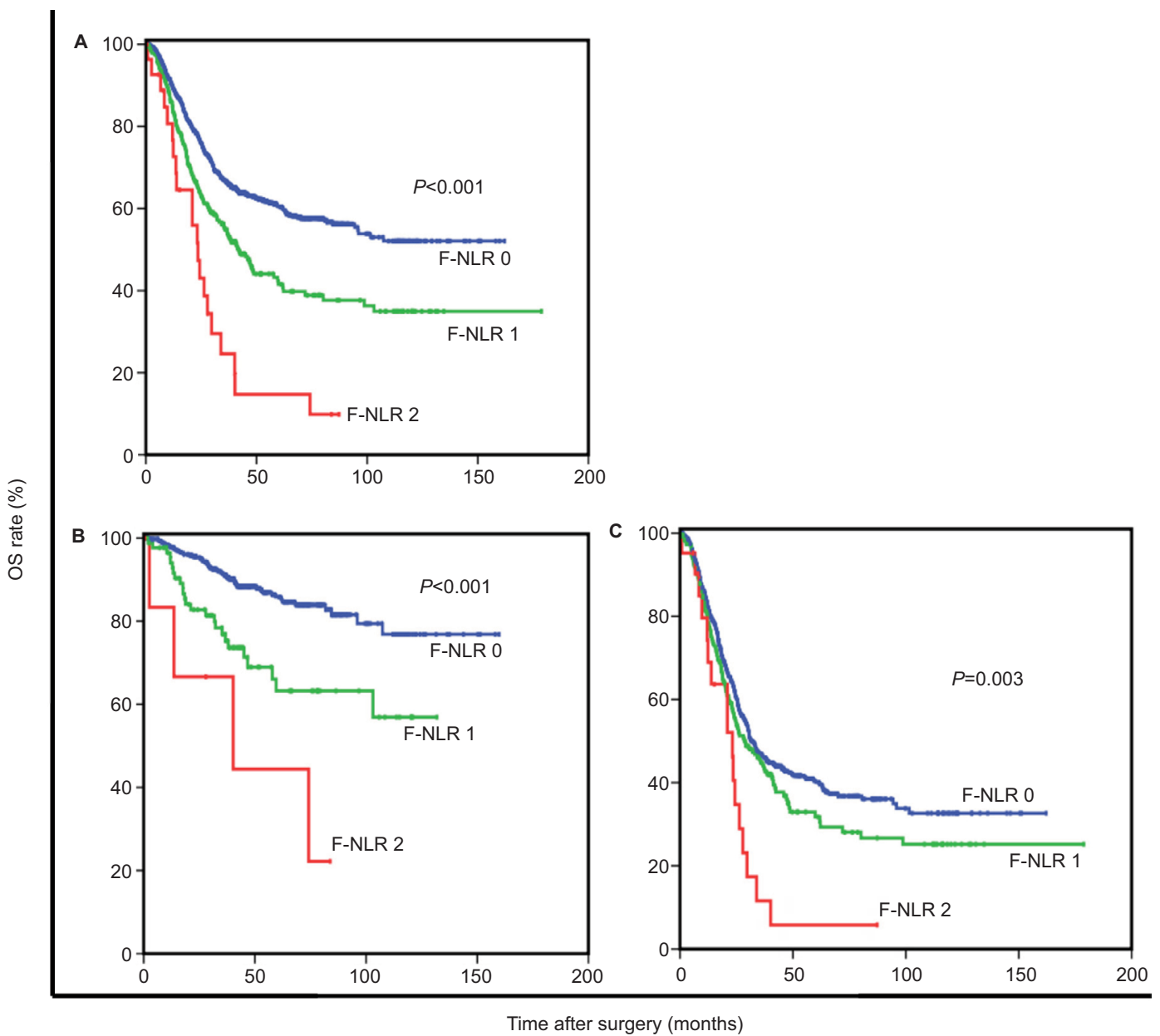

Figure 2 OS based on the F-NLR score in patients with stage I-III (A), stage I-II (B) and stage III (C) GC.

Note: F-NLR, combination of fibrinogen and the NLR.

Abbreviations: GC, gastric cancer; NLR, neutrophil-lymphocyte ratio; OS, overall survival.

F-NLR could identify more high-risk patients than the current TNM staging system alone. Thus, in clinical practice, patients with higher F-NLR scores may need better followup and lower thresholds for early intervention. On the other hand, in patients with F-NLR 2, it might be that preoperative early intervention should be given as a first-line treatment. Further prospective clinical trials are still needed to confirm these results.

Our study has several limitations. First of all, it is a retrospective study including only one institution, which may lead to a selection bias. Second, with limited data availability, some possible confounding factors, such as cardiovascular and kidney disease, probably influence the fibrinogen levels but cannot be analyzed. Third, we lack the follow-up information on disease-free survival, and our conclusions may be strengthened by using other survival measures.

\section{Conclusion}

The F-NLR score is an independent predictor of survival in GC, and it may have important clinical utility for identifying patients at high risk and guiding the administration of individualized treatment. Furthermore, the components of the F-NLR score are objectively measured and routinely available, which makes our findings potentially applicable to clinical practice.

\section{Acknowledgment}

The authors are grateful to the people who kindly cooperated in our study.

\section{Disclosure}

The authors report no conflicts of interest in this work. 


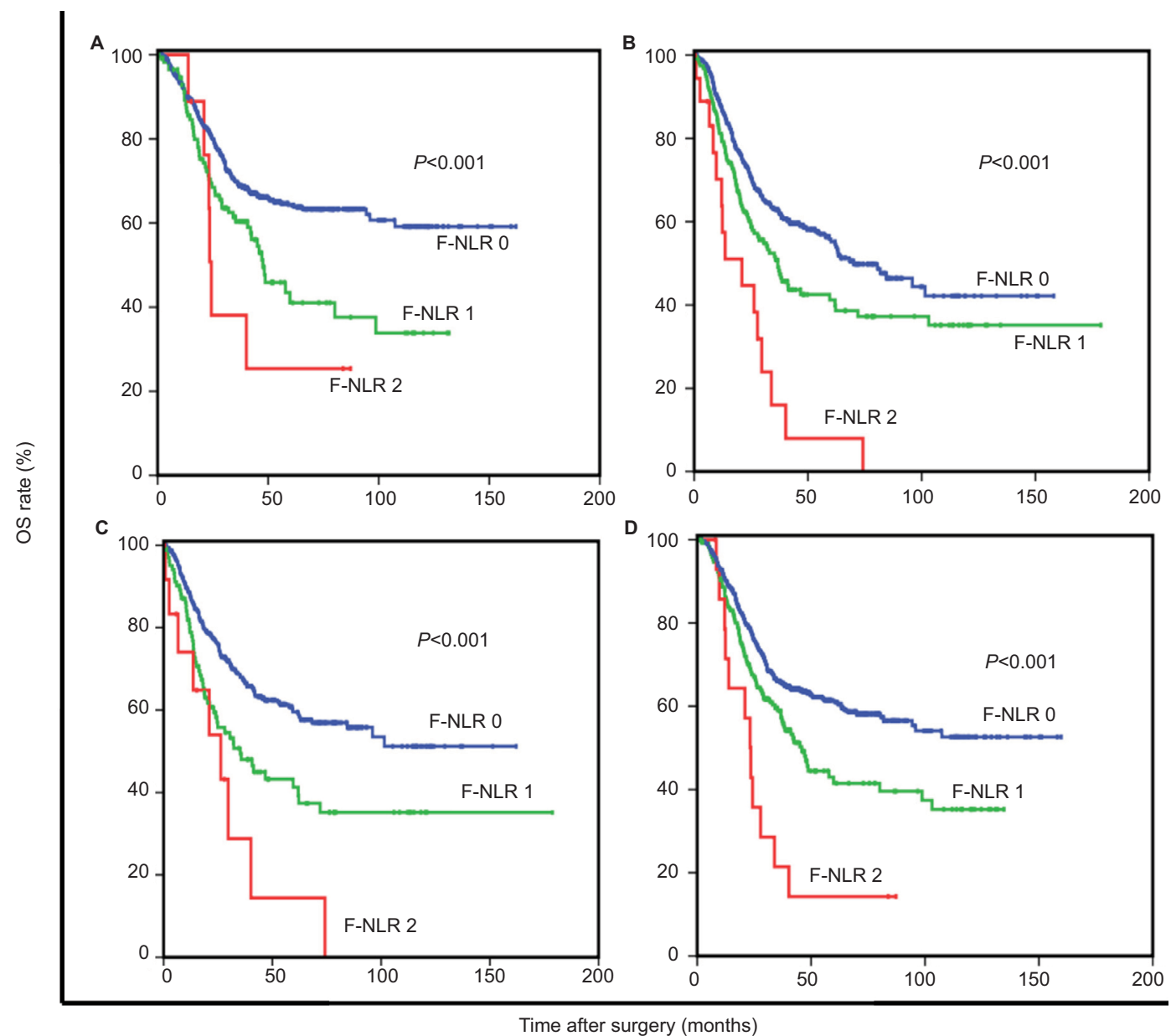

Figure 3 OS based on the F-NLR score in patients aged $<60$ years $(\mathbf{A})$, patients aged $\geq 60$ years $(\mathbf{B})$, patients without adjuvant chemotherapy (C) and patients with adjuvant chemotherapy (D).

Note: F-NLR, combination of fibrinogen and the NLR.

Abbreviations: NLR, neutrophil-lymphocyte ratio; OS, overall survival.

Table 4 Relationships between F-NLR and 5-year OS in patients undergoing curative resection for stage I-III GC

\begin{tabular}{|c|c|c|c|c|c|c|c|c|}
\hline \multirow[t]{2}{*}{ Characteristics } & \multicolumn{2}{|c|}{ TNM stage I } & \multicolumn{2}{|c|}{ TNM stage II } & \multicolumn{2}{|c|}{ TNM stage III } & \multicolumn{2}{|c|}{ TNM stages I-III } \\
\hline & $n$ & 5-year OS & $\mathbf{n}$ & 5-year OS & n & 5-year OS & n & 5-year OS \\
\hline F-NLR & 218 & $92(2)$ & 322 & $74(3)$ & 753 & $37(2)$ & 1293 & $56(2)$ \\
\hline 0 & 195 & $93(2)$ & 249 & $80(3)$ & 542 & $4 I(2)$ & 986 & $61(2)$ \\
\hline I & 23 & 89 (8) & 66 & $53(8)$ & 190 & $32(4)$ & 279 & $42(4)$ \\
\hline 2 & 0 & NA & 7 & $44(2)$ & 21 & $6(6)$ & 28 & $15(8)$ \\
\hline
\end{tabular}

Notes: The values are expressed as $n$ or percentage (standard error). Survival is not calculated if $n \leq 10$. F-NLR, combination of fibrinogen and the NLR.

Abbreviations: GC, gastric cancer; NA, not applicable; NLR, neutrophil-lymphocyte ratio; OS, overall survival.

\section{References}

1. Karimi P, Islami F, Anandasabapathy S, Freedman ND, Kamangar F. Gastric cancer: descriptive epidemiology, risk factors, screening, and prevention. Cancer Epidemiol Biomarkers Prev. 2014;23(5):700-713.

2. Ferlay J, Soerjomataram I, Dikshit R, et al. Cancer incidence and mortality worldwide: sources, methods and major patterns in GLOBOCAN 2012. Int J Cancer. 2015;136(5):E359-E386.

3. Ichikawa D, Komatsu S, Kosuga T, et al. Clinicopathological characteristics of clinical early gastric cancer in the upper-third stomach. World J Gastroenterol. 2015;21(45):12851-12856.
4. Roukos DH. Current advances and changes in treatment strategy may improve survival and quality of life in patients with potentially curable gastric cancer. Ann Surg Oncol. 1999;6(1):46-56.

5. Cuschieri A, Weeden S, Fielding J, et al. Patient survival after D1 and D2 resections for gastric cancer: long-term results of the MRC randomized surgical trial. Surgical Co-operative Group. Br J Cancer. 1999;79(9-10):1522-1530.

6. Piciucchi M, Stigliano S, Archibugi L, et al. The neutrophil/lymphocyte ratio at diagnosis is significantly associated with survival in metastatic pancreatic cancer patients. Int J Mol Sci. 2017;18(4):730. 
7. Kitano Y, Yamashita YI, Yamamura K, et al. Effects of preoperative neutrophil-to-lymphocyte and platelet-to-lymphocyte ratios on survival in patients with extrahepatic cholangiocarcinoma. Anticancer Res. 2017;37:3229-3237.

8. Yodying H, Matsuda A, Miyashita M, et al. Prognostic significance of neutrophil-to-lymphocyte ratio and platelet-to-lymphocyte ratio in oncologic outcomes of esophageal cancer: a systematic review and meta-analysis. Ann Surg Oncol. 2016;23(2):646-654.

9. Zhang X, Zhang W, Feng LJ. Prognostic significance of neutrophil lymphocyte ratio in patients with gastric cancer: a meta-analysis. PLoS One. 2014;9(11):e111906.

10. Tennent GA, Brennan SO, Stangou AJ, O’Grady J, Hawkins PN, Pepys MB. Human plasma fibrinogen is synthesized in the liver. Blood. 2007;109:1971-1974.

11. Perisanidis C, Psyrri A, Cohen EE, et al. Prognostic role of pretreatment plasma fibrinogen in patients with solid tumors: A systematic review and meta-analysis. Cancer Treat Rev. 2015;41:960-970.

12. Tang L, Liu K, Wang J, Wang C, Zhao P, Liu J. High preoperative plasma fibrinogen levels are associated with distant metastases and impaired prognosis after curative resection in patients with colorectal cancer. J Surg Oncol. 2010;102(5):428-432.

13. Shu Y-J, Weng H, Bao R-F, et al. Clinical and prognostic significance of preoperative plasma hyperfibrinogenemia in gallbladder cancer patients following surgical resection: a retrospective and in vitro study. $B M C$ Cancer. 2014;14(1):566.

14. Kijima T, Arigami T, Uchikado Y, et al. Combined fibrinogen and neutrophil-lymphocyte ratio as a prognostic marker of advanced esophageal squamous cell carcinoma. Cancer Sci. 2017;108:193-199.

15. Arigami T, Okumura H, Matsumoto M, et al. Analysis of the fibrinogen and neutrophil-lymphocyte ratio in esophageal squamous cell carcinoma: a promising blood marker of tumor progression and prognosis. Medicine. 2015;94(42):e1702.

16. Zhang X. Long Q. Elevated serum plasma fibrinogen is associated with advanced tumor stage and poor survival in hepatocellular carcinoma patients. Medicine. 2017;96:e6694.

17. Mei Y, Zhao S, Lu X, Liu H, Li X, Ma R. Clinical and prognostic significance of preoperative plasma fibrinogen levels in patients with operable breast cancer. PLoS One. 2016;11(1):e0146233.

18. Wang DS, Ren C, Qiu MZ, et al. Comparison of the prognostic value of various preoperative inflammation-based factors in patients with stage III gastric cancer. Tumour Biol. 2012;33(3):749-756.

19. Aurello P, Tierno SM, Berardi G, et al. Value of preoperative inflammation-based prognostic scores in predicting overall survival and disease-free survival in patients with gastric cancer. Ann Surg Oncol. 2014;21(6):1998-2004.

20. Leitch EF, Chakrabarti M, Crozier JE, et al. Comparison of the prognostic value of selected markers of the systemic inflammatory response in patients with colorectal cancer. Br J Cancer. 2007;97(9):1266-1270.
21. Neal CP, Cairns V, Jones MJ, et al. Prognostic performance of inflammation-based prognostic indices in patients with resectable colorectal liver metastases. Med Oncol. 2015;32(5):144.

22. Colotta F, Allavena P, Sica A, Garlanda C, Mantovani A. Cancer-related inflammation, the seventh hallmark of cancer: links to genetic instability. Carcinogenesis. 2009;30(7):1073-1081.

23. Mantovani A, Garlanda C, Allavena P. Molecular pathways and targets in cancer-related inflammation. Ann Med. 2010;42(3):161-170.

24. Ku JH, Kang M, Kim HS, Jeong CW, Kwak C, Kim HH. The prognostic value of pretreatment of systemic inflammatory responses in patients with urothelial carcinoma undergoing radical cystectomy. Br J Cancer. 2015;112(3):461-467.

25. Hutterer GC, Sobolev N, Ehrlich GC, et al. Pretreatment lymphocytemonocyte ratio as a potential prognostic factor in a cohort of patients with upper tract urothelial carcinoma. J Clin Pathol. 2015;68(5):351-355.

26. Grenader T, Waddell T, Peckitt C, et al. Prognostic value of neutrophilto-lymphocyte ratio in advanced oesophago-gastric cancer: exploratory analysis of the REAL-2 trial. Ann Oncol. 2016;27(4):687-692.

27. Zhang J, Zhang H-Y, Li J, Shao X-Y, Zhang C-X. The elevated NLR, PLR and PLT may predict the prognosis of patients with colorectal cancer: a systematic review and meta-analysis. Oncotarget. 2017;8(40):68837-68846.

28. Chang $\mathrm{PH}$, Wang $\mathrm{CH}$, Chen EY, et al. Glasgow prognostic score after concurrent chemoradiotherapy is a prognostic factor in advanced head and neck cancer. Chin J Cancer Res. 2017;29:172-178.

29. Yamamoto M, Kurokawa Y, Miyazaki Y, et al. Usefulness of Preoperative Plasma Fibrinogen Versus Other Prognostic Markers for Predicting Gastric Cancer Recurrence. World J Surg. 2016;40(8):1904-1909.

30. Luo Y, Kim HS, Kim M, Lee M, Song YS. Elevated plasma fibrinogen levels and prognosis of epithelial ovarian cancer: a cohort study and meta-analysis. J Gynecol Oncol. 2017;28(3):e36.

31. Simpson-Haidaris PJ, Rybarczyk B. Tumors and fibrinogen. The role of fibrinogen as an extracellular matrix protein. Ann NY Acad Sci. 2001;936:406-425.

32. Palumbo JS, Talmage KE, Massari JV, et al. Platelets and fibrin(ogen) increase metastatic potential by impeding natural killer cell-mediated elimination of tumor cells. Blood. 2005;105:178-185.

33. Ridker PM, Howard CP, Walter V, et al. Effects of interleukin-1beta inhibition with canakinumab on hemoglobin A1c, lipids, C-reactive protein, interleukin-6, and fibrinogen: a phase IIb randomized, placebocontrolled trial. Circulation. 2012;126:2739-2748.

34. Zhang D, Zhou X, Bao W, et al. Plasma fibrinogen levels are correlated with postoperative distant metastasis and prognosis in esophageal squamous cell carcinoma. Oncotarget. 2015;6(35):38410-38420.

35. Arigami T, Uenosono Y, Ishigami S, et al. A novel scoring system based on fibrinogen and the neutrophil-lymphocyte ratio as a predictor of chemotherapy response and prognosis in patients with advanced gastric cancer. Oncology. 2016;90(4):186-192.
Cancer Management and Research

\section{Publish your work in this journal}

Cancer Management and Research is an international, peer-reviewed open access journal focusing on cancer research and the optimal use of preventative and integrated treatment interventions to achieve improved outcomes, enhanced survival and quality of life for the cancer patient. The manuscript management system is completely online and includes
Dovepress

a very quick and fair peer-review system, which is all easy to use. Visit $\mathrm{http}: / / \mathrm{www}$.dovepress.com/testimonials.php to read real quotes from published authors. 